\title{
Nanocalorimetric platform for accurate thermochemical studies in microliter volumes
}

\author{
R. Padovani, T. Lehnert, R. Trouillon and Martin A.M. Gijs*
}

A straightforward and general way for monitoring chemical reactions is via their thermal signature. Such approach requires however an experimental setup with a high thermal stability that simultaneously allows time-resolved heat detection with high sensitivity. We present a nanocalorimetric platform for accurate thermochemical studies of (bio-)chemical reactions in a miniaturized format (tens of microliter volume), characterized by a fast thermalization time to a preset temperature ( $<30$ minutes), an excellent base temperature stability $( \pm 1 \mathrm{mK}$ ) and a fast sensing response time (few seconds). The platform is built around a commercial thermopile-based sensor chip, on which an open-well reservoir holding the sample is directly positioned. The sample is, prior to the experiment, pipetted into the reservoir, in which small aliquots of reagents are injected subsequently and sequentially via thermalized microfluidic conducts. The design of the platform is optimized by means of numerical simulations. Via thermoelectric calibration using a resistive heater positioned either on the sensor chip or in the reservoir, we obtain a maximum power sensitivity of $2.7 \mathrm{~V} / \mathrm{W}$ and a heat limit of detection of $70 \mathrm{nW}$. The excellent functionality of the platform is demonstrated by measuring the reaction enthalpy of 1-propanol in water and the rate constant $k$ and enthalpy change of the oxidation reaction of glucose catalyzed by glucose oxidase, showing good agreement with literature data. Our versatile platform may be applied to many thermochemical studies, including thermodynamic analysis and kinetic reaction analysis, and its ease of use will allow implementation of many different experimental protocols.

\section{Introduction}

Calorimetry is a powerful analytical method for thermochemical characterization of physical, chemical or biological processes. Its working principle is based on the transduction of heat into an electrical signal. It is a very versatile method that is suitable for a variety of applications, provided that the chemical reaction process involves an enthalpy variation[1]. In particular, in life science research, calorimetry is considered the gold standard for studying biomolecular interactions, as it allows both the full thermodynamic profiling, including the quantification of enthalpy, free energy and entropy[2] and the kinetic analysis of bioprocesses[3]-[5]. Furthermore, this technique has the unique capability of measuring directly the metabolic heat produced by living organisms[6], [7], enabling applications in disease diagnosis, drug development, toxicology, and environmental testing[8], [9]. The advantages of calorimetry are many: (i) it is a label-free technique, which does not require specific prior sample preparation, (ii) it is non-invasive, as it does not alter the nature of the sample allowing further analyses, and (iii) it is not limited by sample opacity, in contrary to the use of optical characterization methods[1], [10]. However, the omnipresence of thermo-chemical phenomena in nature qualifies heat as a non-specific signal, which may become a disadvantage if experiments are not carefully designed. Indeed, unwanted reactions, nonspecific molecular binding events, and evaporation may cause misleading interpretation of the experimental data[1], [7], [11]. The main drawbacks of classical calorimetry are the requirement for relatively large sample volumes (at least a few $\mathrm{ml}$ ) to achieve a large heat signal that allows detection with sufficient sensitivity, equipment cost and inflexibility in terms of its application to living organism studies and in terms of high-throughput implementation[12].

The miniaturized version of calorimetry, often named nanocalorimetry, uses volumes in the $\mathrm{nl}-\mu \mathrm{l}$ range, resulting in proportionally smaller heat signals, but benefiting from the existence of extremely sensitive miniaturized sensors, enabling detection in the $\mathrm{nW}$ range or below[13]. The versatility of nanocalorimetry has now been demonstrated by a wide range of applications that are documented in literature[14]. The great majority of nanocalorimeters relies on the use of a thermopile sensor, which is microfabricated on a thin membrane with low heat capacity that is suspended in a thicker frame. This configuration guarantees a high thermal insulation and enables the detection of 
heat produced by small sample volumes[12], [14]. Thermopile-based sensors for liquid applications are commercially available[15], but they have been also custom-built[16]-[20], driven by particular experimental designs. Also, thermistor-based nanocalorimeters have been developed[5], [21]-[23].

Nanocalorimeters may be classified into two different types: batch-mode, also called open-chamber systems, and flow-through-mode, also called closed-chamber systems. In open-chamber nanocalorimeters, the sample is dispensed directly into a microwell that is placed on the sensor chip[17], [24]-[27]. For instance, Johannessen et al. demonstrated the feasibility of monitoring heat production by a very small number of mammalian cells[17], [28] in sample volumes of hundreds of $\mathrm{pl}$. In a closed-chamber device, the sample volume is essentially confined, but there is still a fluidic connection of this volume to the outside world with microchannels allowing sample and reagent delivery as well as drain of the reactants and this in a dynamic fashion. Such systems clearly are well adapted for integration and automation of a measurement protocol[10], [18]-[20], [29]-[31]. However, the same fluidic connections may introduce additional heat loss paths and worsen the thermal insulation to the environment, thus affecting the sensitivity[14], [18]. Lee et al. developed a device based on a parylene-polymer microfluidic calorimeter chip combined with soft-polymer fluidic components[18]. On-chip vacuum encapsulation was used to improve thermal insulation, and $4.2 \mathrm{nW}$ resolution was demonstrated for $\mathrm{nl}$ sample volumes. The investigation of slow metabolic processes require sample volumes of at least a few $\mu \mathrm{l}$, as the signal to noise ratio is directly proportional to sample volume. For these applications, the precision of the temperature stabilization is particularly critical for obtaining a good signal resolution[32]. A miniaturized flow-through calorimeter was therefore installed in a sophisticated thermostat system, providing a temperature stability of better than $40 \mu \mathrm{K}[10]$, [13], [16], [33][36]. More recently, such system was combined with the so-called segmented flow technology, which consists in repetitive sequential processing of sample segments separated by immiscible inert segments[37], [38].

In this work, we report the development and characterization of a novel nanocalorimetric platform for the accurate study of the heat that is generated by (bio-)chemical reactions using a small sample reservoir of a few tens of $\mu$ l. In particular, applications in the field of biochemistry and molecular biology have been addressed, often requiring simultaneously both relatively large sample volumes (of the order of a few tens of $\mu$ ) and precision temperature stabilization. Our system has the advantages of an open-chamber configuration, as initially the sample is simply pipetted in a reservoir that was priorly mounted on a commercial thermopile sensor chip. Hereafter, the reservoir is confined by embedding it into an isothermal sample holder that also provides fluidic connections to the sample via the sidewalls of the reservoir. This allows profiting from the advantages of a flow-through system, as, after thermal stabilization, additional reagents may be easily sequentially injected. The accessibility of the reservoir prior and during the experiment is crucial for the versatility of the platform. In fact, the modularity of the system allows applying sample preparation steps to the reservoir that are independent from the nanocalorimetric measurements themselves, which will be particularly useful for biological samples. Also, during the calorimetric measurements additional reagents may be injected without perturbing the thermalization of the system. Particular attention was given to the platform design which was guided by computational thermal simulations. This approach allowed optimizing two distinctive thermal time constants for the system, namely (i) a time constant for reaching a stable platform setpoint temperature, thereby avoiding long stabilization times, and (ii) a time constant of the sensing system for a good time-resolved detection of heat generation/consumption processes. The platform had a very high temperature stability (down to $\pm 1 \mathrm{mK}$ over a period of about one hour for a setpoint temperature of $25^{\circ} \mathrm{C}$ ), and its excellent functionality was demonstrated by a thermodynamic study of the mixing enthalpy of 1-propanol in water and by a kinetic study of the glucose oxidase-catalyzed reaction of glucose. 


\section{Experimental}

\section{System description}

The development of the nanocalorimetric platform required both an accurate mechanical and thermal design, and fine-tuning of the temperature control system. In particular, the first requirement to be met is the ability to adjust a specific setpoint temperature and to minimize thermal gradients inside the isothermal holder, in particular around the sensing area. As the sensing principle is based on detecting thermal gradients originating from heat generated by a chemical or biological sample, any thermal gradient in absence of the sample would result in an undesired background signal. A second stringent requirement is the temperature stability of the whole isothermal holder, which serves in particular as heat sink for the sensor chip. Even minor fluctuations of the temperature of the heat sink may generate fluctuations in the temperature gradients over the sensing area and, thus, instabilities in the background signal. For highly sensitive heat power measurements, i.e. below the $\mathrm{mW}$ range, heat sink temperature control and stability must be optimized.

Moreover, it is important that heat generated by the sample is conducted only through the sensing area on the chip. The interface between the different components is critical in order to minimize any heat loss through alternative thermal paths. Finally, the different thermal time constants of the platform have to be considered in the design. On the one hand, the platform temperature has to reach steady state temperature in a reasonable time window (i.e. less than an hour), so its volume and thermal inertia should be kept small. On the other hand, reducing the thermal inertia also reduces damping of ambient temperature fluctuations. The second critical time constant relates to the time response of the sensor with respect to heat generated by the sample: it should be fast enough to detect dynamic events related to (bio-)chemical processes.

The isothermal holder, developed according to these requirements is sketched in Fig. 1A. It is composed of the following elements: a thermopile sensor (only the outer ceramic frame is visible in the figure), a polycarbonate (PC) sample reservoir, three aluminum parts assembled around the sensor, whose temperature is controlled by a closed loop Proportional-Integral-Derivative (PID) control system and two poly(methylmethacrylate) (PMMA) plates (bottom and top of the holder). An outer PMMA box is also used to provide additional thermal insulation with respect to ambient temperature fluctuations (not included in the sketch).

\section{The thermopile sensor and working principle}

The sensor is a commercially available thermopile chip (XEN-NCM 9924, Xensor Integration). The aluminum-silicon thermopile is fabricated on a $22 \mu \mathrm{m}$ thick monocrystalline silicon membrane (area of $8.3 \times 8.3 \mathrm{~mm}^{2}$ ) which is sitting on a thicker silicon rim. The sensor chip is shown in Fig. 1B. The thermopile is made of a series of thermocouples which are radially distributed around a central square area of about $4 \times 4 \mathrm{~mm}^{2}$ : the hot junctions of the thermopile, which define the sensing area, are located on the inner part of the membrane, while the cold junctions are in thermal contact with the rim. The working principle of the thermopile sensor is based on the thermoelectric conversion of the thermal gradient present between hot and cold junctions of the thermopile into a voltage $V_{T P}$. In particular, the thermal gradient is caused by the production/absorption of heat by the sample $\left(Q_{\text {SAMPLE }}\right)$ in the sensing area, and the heat flow from the sample towards the heat sink is determined by the membrane thermal resistance. Thus, in order to use this sensing mechanism it is crucial to keep the cold junctions at a constant temperature and to minimize thermal gradients in the device not related to the sample heat production. Using the serpentine aluminum thin film heater integrated on the central area of the membrane (see Fig. 1B) is the most direct method for electrical calibration of the thermopile sensor, avoiding most effects of heat loss through other thermal pathways than the sensor membrane. 


\section{The isothermal holder and the temperature control system}

The sensor chip is incorporated into an isothermal holder that provides a temperature regulated environment (external dimensions of $60 \times 60 \times 30 \mathrm{~mm}^{3}$ ). As depicted in Fig. 1A, the silicon sensor rim of the sensor chip is tightly clamped between two aluminum parts to provide a good thermal contact. A temperature controller (Model 335, Lake Shore Cryotronics, Inc.) which is operated in a closed loop PID mode, is used to stabilize the temperature of the isothermal holder at the setpoint value. The temperature control system includes also a temperature sensor (named PID sensor) and an electrical heater (PID heater). The PID sensor is a miniature platinum sensor (1.6 $\times 1.2$ $\mathrm{mm}^{2}$ ) with $1000 \Omega$ nominal resistance at $0^{\circ} \mathrm{C}$ (Pt1000 class A, IST Innovative Sensor Technology). A small groove in the aluminum part that is in direct contact with the sensor chip accommodates the PID sensor. Good thermal contact is achieved by clamping the sensor with an aluminum plate ( $1 \mathrm{~mm}$ thick). The PID-controlled heater is a flat polyimide square heater $\left(50.8 \times 50.8 \mathrm{~mm}^{2}, 36.1 \Omega\right.$ resistance, Minco) which is fixed on top of the aluminum plate by a PMMA plate ( $3 \mathrm{~mm}$ thick). The PMMA plate also reduces heat loss to the external environment. The position of the two PID controlling elements was chosen carefully in order to guarantee accurate temperature reading, which should ideally correspond to the temperature of the cold junctions, and to allow a fast response of the PID controller to minimize temperature fluctuations.

\section{Sample reservoir and sample injection system}

The sample reservoir is made of a $7 \times 7 \mathrm{~mm}^{2} \mathrm{PC}$ well with an inner size of $5 \times 5 \mathrm{~mm}^{2}$, which is glued on the bottom to a $25 \mu \mathrm{m}$ thick polystyrene membrane (Goodfellow Cambridge Ltd.), as shown in Fig. 1C. The reservoir is positioned on the sensor membrane so that the sensor is not contaminated by the sample and may be reused for several experiments. Minimizing the thermal resistance between sample and the sensor membrane is crucial for high-sensitive heat detection. Therefore a thin film of thermal grease (Thermigrease TG 20025, Müller GmbH) is applied to the interface of the two surfaces. Fluidic connections to the reservoir are provided by polytetrafluoroethylene (PTFE) thin tubings $(0.3 \times 0.76 \mathrm{~mm}$ PTFE microtube, Fisher Scientific) that are inserted through the fluidic connection fabricated into the isothermal holder and in the side walls of the reservoir. As the tubing is in direct contact with the isothermal holder and is filled with the reagents at the beginning of each experiment, the sample is thermalized prior to its injection, minimizing thermal injection artefacts.

\section{Platform automation and data acquisition}

A software-controlled syringe pump (Nemesys Syringe Pump, Cetoni $\mathrm{GmbH}$ ) is used for automated sample injection of typically 1.5 to $5 \mu \mathrm{l}$. As sketched in Fig.1D, all other equipments are controlled through a custom-made program developed in Labview (National Instruments), including the temperature controller, a nanovoltmeter (34420A, Agilent) for thermopile signal acquisition, and a sourcemeter (2400, Keithley Instruments) for implementing the calibration procedure.

\section{Materials used for (bio-)chemical experiments}

The validation of this platform is based on the measurement of the heat released/absorbed during different chemical reactions. The first reaction tested is the mixing of 1-propanol (1-propanol anhydrous, 99.7\%, SigmaAldrich) in deionized water (DIW). The second reaction is the oxidation of glucose (D-Glucose $\geq 99.5 \%$, SigmaAldrich) catalyzed by glucose oxidase (Glucose Oxidase from Aspergillus niger 100,000-250,000 units/g, SigmaAldrich). In this second case, phosphate buffered saline (PBS pH 7.4 Gibco, Life Technologies) was used as the buffer solution. 


\section{Results and discussion}

\section{Numerical simulation of the system and its experimental thermal stability}

Simulations have been carried out in Comsol Multiphysics, in order to model the heat transfer phenomena in the isothermal holder. Detailed results are reported in the Supporting Information section. In particular, our studies allowed to optimize the system with (i) a time constant $\tau^{\prime}$ of $40 \mathrm{~min}$ to both reach a stable platform temperature and minimize the thermal gradient over the sensing area, and (ii) a time constant $\tau^{\prime \prime}$ of $8 \mathrm{sec}$ to obtain a stable thermal gradient between hot and cold junctions of the thermopile in response to heat generated by the sample.

Results of experimental tests, aiming at both evaluating the temperature stability of the platform and estimating the background signal, are also reported in the Supporting Information section. In particular, the thermalization waiting time was reduced to 10-15 minutes to reach the setpoint temperature, and in total to 20-25 minutes to reach the maximum temperature stability. The temperature stability $\sigma_{T}$ is estimated to be $\pm 1 \mathrm{mK}$, which is the maximum stability reachable for the temperature controller in use, and the corresponding voltage signal stability $\sigma_{V}$ is $\pm 80 \mathrm{nV}$.

\section{Thermo-electrical calibration and limit of detection}

For calibrating the nanocalorimetric platform, the power sensitivity coefficient $P S=V_{T P} / P_{R}$, i.e. the conversion coefficient that relates the thermopile voltage signal $V_{T P}$ to the applied heat power $P_{R}$ has to be determined for different experimental configurations. Two different configurations, schematically shown in Fig. $2 \mathrm{~A}$ have been considered. The first one uses the resistive heater integrated in the silicon chip membrane ( $R \_I N T$, no reservoir), while the second one uses an external resistive heater $\left(R_{-} E X T\right)$, which is a miniaturized $100 \mathrm{k} \Omega$ thermistor (MicroBetaCHIP Thermistor Probe, Measurement Specialties) immersed into the reservoir filled with $50 \mu \mathrm{DIW}$. The second calibration procedure mimics more closely the real experimental situation, i.e. heat generation by a liquid sample in the reservoir that is placed on the sensor membrane. In both cases, periodically increasing electrical power was applied to the resistive heaters, which is converted by the Joule effect into heat power $\left(P_{R_{-} I N T}\right.$ and $P_{R_{-} E X T}$ for the integrated and external heater, respectively) and the corresponding thermopile voltage $V_{T P}$ is measured. This sequence is shown for the integrated heater in Fig. 2B. The calibration curves for the thermopile voltage $V_{T P}$ as a function of the heat power generated by the resistive heater $P_{R}$ for both configurations are presented in Fig. $2 \mathrm{C}$. The corresponding power sensitivity coefficients can be determined as $P S_{R_{-} I N T}=2.7 \mathrm{~V} / \mathrm{W}$ and $P S_{R_{-} E X T}=1.9 \mathrm{~V} / \mathrm{W}$, for the internal and external heater, respectively. The power sensitivity using the integrated heater is higher, as the heating element and the sensing element are integrated on the same silicon membrane resulting in very efficient heat power to voltage conversion with minimum heat loss. When including a reservoir with an external heater heat losses through alternative thermal paths arise, thus reducing the power sensitivity of the system. The inset in Fig. $2 \mathrm{C}$ shows the calibration curves for very small heat power $(0-300 \mathrm{nW})$, from which we determine a limit of detection (LOD) of the platform of $70 \mathrm{nW}$ for the integrated heater, and a LOD of about $170 \mathrm{nW}$ for the external heater (corresponding to $\pm 3 \sigma_{P}$ where $\sigma_{P}=P S \times \sigma_{V}$ is the standard deviation of the heat power signal, and $\sigma_{V}$ is the standard deviation of the thermopile voltage $\left.V_{T P}\right)$. The first value can be considered as the ultimate intrinsic limit of detection of the platform, whereas the second value corresponds better to a real experimental situation.

\section{Heat of mixing of 1-propanol in water}

As already pointed out by Wadsö et al.[39], calibration based on electrically generated heat may cause systematic errors as the induced thermal gradients may not fully reflect the experimental conditions of heat-generating (bio)chemical reactions. For this reason, a test reaction having well-known thermochemical properties is required to further validate the calibration and the sensing system. 
The mixing of 1-propanol in water at $25{ }^{\circ} \mathrm{C}$ was chosen here for this purpose, as data for the molar enthalpy of mixing, or molar excess enthalpy $H^{E}$, of 1-propanol in water are available in literature[39], [40]. Data reported by Davis et al.[40] were taken as a reference and are summarized in Fig. 3A. The differential molar excess enthalpy $d H^{E} / d X$, plotted on the same figure, was obtained by differentiating $H^{E}$ with respect to $X$, where $X$ is the mole fraction of 1-propanol in water.

In order to validate the functionality of the nanocalorimetric platform, differential molar excess enthalpy $d H^{E} / d X$ values obtained with this platform were compared with literature data. The experimental procedure consisted in prefilling the reservoir with $45 \mu \mathrm{l}$ of 1-propanol in water solutions at different initial concentrations. The inlet tubing connecting the reservoir was filled with $5 \mu \mathrm{l}$ of pure 1-propanol. After allowing a thermalization time of $20 \mathrm{~min}$ to reach the setpoint temperature of $25^{\circ} \mathrm{C}, 5 \mu \mathrm{l}$ of pure 1-propanol were injected in the reservoir, resulting in a total volume of $50 \mu \mathrm{l}$. As the inlet tube passes through the metal block of the platform, the injected volume is well thermalized at $25^{\circ} \mathrm{C}$. As a result, a single experiment may last less than 30 minutes, which is advantageous when planning many sequential and independent experiments. Additionally, an alternative experimental protocol was also tested, and it described more in detail in the Supporting Information section: it consisted in applying up to three sequential injections of $5 \mu \mathrm{l}$ of pure 1-propanol in the same reservoir prefilled with $45 \mu$ l of 1-propanol in water solution. This second approach allows to carry on tests at increasing concentrations of reactant in an even more time-efficient manner.

The results of a series of experiments using an initial concentration of 1-propanol in water from $0 \%$ to $89 \%(\mathrm{v} / \mathrm{v})$ is shown in Fig. 3B. The curves show the heat power generated upon mixing corresponding to the total enthalpy change over time $d H_{T O T}^{E} / d X$. In order to compare with literature values, some data processing is required to calculate the corresponding differential molar excess enthalpy $d H^{E} / d X$, as summarized in Fig. $3 C$. The following steps were applied: (i) normalizing the total differential excess enthalpy $d H_{T O T}^{E_{T}} / d X$ by the number of moles in solution in order to obtain the corresponding molar value which is $d H^{E} / d X$; (ii) integrating with respect to time to obtain the excess molar enthalpy $H^{E}$, which is the molar enthalpy change measured when the solution concentration varies from the initial mole fraction $X_{i}$ to the final mole fraction $X_{f}$; (iii) dividing by the mole fraction difference $\left(X_{f}-X_{i}\right)$ to obtain the differential molar excess enthalpy $d H^{E} / d X$. Being a discrete derivative obtained from the experimental measurements, the central mole fraction $X_{c}=X_{i}+\left(X_{f}-X_{i}\right) / 2$ has been calculated in order to plot pairs of values $\left(X_{c}\right.$, $\left.d H^{E} / d X\right)$ on the $d H^{E} / d X$ vs $X$ curve. Fig. 3D shows the comparison between the thus calculated data points and the differential molar excess enthalpy curve derived from literature and clearly demonstrates a good match. A power sensitivity coefficient $P S_{\text {exp }}$ of $0.6 \mathrm{~V} / \mathrm{W}$ was used, which is a value that is about 3 times lower than the conversion coefficient $P S_{R_{-} E X T}$ obtained with the external heater. This fact underlines the importance of system calibration using chemical reactions, which generate a heat signal that physically is originating from a distance further away from the sensor membrane than when using a resistive heater.

\section{Enzymatic study of glucose oxidation, catalyzed by glucose oxidase}

The nanocalorimetric platform was also used to study the enzymatic activity of glucose oxidase (GOx) at $25{ }^{\circ} \mathrm{C}$, when catalyzing the oxidation of glucose. Glucose oxidase uses molecular oxygen to catalyze the oxidation of glucose to gluconic acid and the overall reaction can be described as follows[41], [42]: 


$$
\text { glucose }+\mathrm{O}_{2}+\mathrm{H}_{2} \mathrm{O} \stackrel{\text { GOX }}{\longrightarrow} \text { gluconic acid }+\mathrm{H}_{2} \mathrm{O}_{2}
$$

In particular, experiments were performed in presence of dissolved oxygen (DO) under atmospheric conditions, which corresponds to an initial DO concentration of $0.25 \mathrm{mM}[43]$. The experimental procedure for this experiment is very similar to the previously applied protocol, except for the liquid volumes used. The reservoir was filled with $25 \mu \mathrm{l}$ of glucose oxidase solution ( $250 \mathrm{U} / \mathrm{ml}$ in PBS), the tubing was filled with $1.5 \mu \mathrm{l}$ of glucose solution in PBS, the temperature was set to $25^{\circ} \mathrm{C}$ and 30 minutes of thermalization were allowed before injecting the glucose solution. The volume injected was adjusted so that the final glucose concentration ranged from 0 to $40 \mathrm{mmol} / \mathrm{l}$. Recalibration of the platform using 1-propanol in water as test reaction with the reduced sample volume ( 25 $\mu$ instead of $\sim 50$ $\mu l)$ resulted in an improved power sensitivity $P S_{\text {exp }}$ of $1 \mathrm{~V} / \mathrm{W}$. A small sample volume results in heat generation closer to the thermopile sensor, which is more efficiently detected indeed. This power sensitivity coefficient was applied to all glucose oxidation experiments.

Results from a series of tests are presented in Fig. 4A, showing the heat power $P$ released over time when glucose is injected in the glucose oxidase solution. It is possible to observe that the heat power released $P$ corresponds to the heat energy released per unit time, and it is directly proportional to the number of moles of glucose which are consumed per unit time, that is the reaction rate $V$ in $\mathrm{mol} /(\mathrm{l} \cdot \mathrm{s})$ :

$$
P=h \cdot V
$$

where $h$ is the heat energy released per mole of glucose consumed per volume unit $(\mathrm{J} /(\mathrm{mol} / \mathrm{l}))$. As the catalytic activity starts, the heat power measured has the maximum value $\left(P_{0}\right)$, which corresponds to an initial reaction rate $V_{0}$, and then slowly decreases. By integration of the heat power with respect to time, the energy released over time plot is obtained, as shown in Fig. 4B. Additionally, by calculating the initial heat power $P_{0}$ for each glucose concentration, the corresponding power vs concentration plot is obtained and presented in Fig. 4C. For most enzymatic assays, and typically for GOx mediated glucose oxidation, the curve obtained by plotting the initial reaction rate $V_{0}$ as a function of the glucose substrate concentration $[S]$ is described by the Michaelis-Menten model, which is based on the following equation [44]-[46]:

$$
V_{0}=V_{\text {max }} \cdot \frac{[S]}{[S]+K_{M}}
$$

where $V_{\max }$ is the maximal rate attained when all catalytic sites of the enzyme are saturated and $K_{M}$ is the Michaelis constant.

As the heat power $P$ is directly proportional to the reaction rate $V$, a similar behavior is observed when plotting the initial heat power $P_{0}$ as a function of the glucose concentration. Experiments were carried out for different $\mathrm{GOx}$ concentrations and fitting with the Michaelis-Menten-like equation $P_{0}([S])$ was carried out as shown in Fig. 4C (dashed lines; adjusted R-squared higher than 0.97 ) to obtain the two distinctive parameters $P_{\text {MAX }}$ and $K_{M}$. The maximal heat power $P_{\text {MAX }}$ estimated is about $210 \mu \mathrm{W}$ and $230 \mu \mathrm{W}$ for a GOx concentration of $125 \mathrm{U} / \mathrm{ml}$ and 250 $\mathrm{U} / \mathrm{ml}$, respectively. More interestingly, the corresponding Michaelis constants $K_{M}$ are about 50 and $25 \mathrm{mmol} / \mathrm{l}$, which are very close to reported values in literature[47]-[49]. Considering the curve for the GOx concentration of $250 \mathrm{U} / \mathrm{ml}$, it is possible to observe the linear region of the Michaelis-Menten plot for low glucose concentration ([glucose] $\ll 25 \mathrm{mmol} / \mathrm{l}$ ), which is typical of a first order enzyme-substrate reaction, before enzyme saturation becomes important. Fig. 4D shows a magnification in the $0-5 \mathrm{mmol} / \mathrm{l}$ range of glucose concentrations: in this range of glucose concentrations, the heat power $P_{0}$ and, thus, the reaction rate $V_{0}$ is directly proportional to the initial substrate concentration [ $\left.S_{0}\right]$. A linear fitting (dashed line; adjusted R-squared equal to 0.96 ) allows the estimation of the slope of the fitted line $\alpha$ according to the following equation: 


$$
P_{0}=\alpha \cdot\left[S_{0}\right]
$$

The estimated value of $\alpha$ from the experimental data is $6.68 \mu \mathrm{W} /(\mathrm{mmol} / \mathrm{l})$. Furthermore, for this type of reactions, the substrate concentration $[S]$ decays exponentially over time as:

$$
[S]=\left[S_{0}\right] e^{-k \cdot t}
$$

where $k$ is the rate constant (1/s). Thus, from equation (1) and (4) it is possible to deduce that the reaction rate and, consequently, the heat power released decay exponentially too, as:

$$
P=h \cdot V=h \cdot \frac{d[S]}{d t}=-h \cdot k \cdot[S]=-h \cdot k\left[S_{0}\right] e^{-k \cdot t}
$$

Interestingly it is possible to estimate the rate constant $k$ by fitting the experimental data shown in Fig. 4A, highlighted by the dashed rectangle. In particular, a short duration after the power peak was chosen to guarantee that the system was still in the conditions close to its initial status and that the first order reaction obtained from the simplification of Michaelis-Menten is still a good approximation. From the exponential fitting done over a time window of 2 minutes after the maximal peak, for the glucose concentration range from 1.3 to $5 \mathrm{mmol} / \mathrm{l}$ ([glucose] $<<25 \mathrm{mmol} / \mathrm{l}$ ), the estimated average rate constant $k$ is $0.00221 / \mathrm{s}$ (adjusted R-squared higher than 0.80 ). Finally, by applying equation (5) to the time instant of the maximal peak $P_{0}$ (considered as the onset of the reaction, once the system has overcome its mass transport limitation) and knowing the proportionality coefficient $\alpha$ from equation (3), the following relation can be deduced:

$$
P_{0}=-h \cdot k\left[S_{0}\right]=\alpha\left[S_{0}\right]
$$

As the rate constant $k$ and the proportionality coefficient $\alpha$ have been estimated, it is possible to calculate $h$ which, divided by the sample volume $v$ of $26.5 \mu$, gives the information on the energy released during the reaction per unit of mole, that is the enthalpy change. The enthalpy change estimated with this procedure is $-115 \pm 15 \mathrm{~kJ} / \mathrm{mol}$ (for the $250 \mathrm{U} / \mathrm{ml} \mathrm{GOx}$ solution), which is in good agreement with values available from literature[48].

A posteriori analysis of possible limiting effects of dissolved oxygen depletion, which were documented in literature[48], [50], has also been carried out. Such limitation was not observed experimentally in our case and the results were indeed in agreement with literature on the Michaelis-Menten model[46], [48]. Oxygen diffusion from the top surface of the liquid to the bottom of the reservoir may take up to 4 minutes, if we consider the inner size of the reservoir of $5 \times 5 \mathrm{~mm}^{2}$, the sample volume of $25 \mu \mathrm{l}$, and thus a mean diffusive path of up to $1 \mathrm{~mm}$ (a diffusion coefficient $D$ of oxygen in water of $2 \cdot 10-5 \mathrm{~cm}^{2} / \mathrm{s}$ was taken[51] and the mean diffusive time $t$ calculated as $x^{2} /(2$. D)). Therefore, re-supply of oxygen over the duration of the experiment is continuously enabled. Moreover, the highest consumption of glucose per minute, which is observed at the maximum reaction rate, may be estimated by dividing the heat power measured (Fig. 4A) by the reaction enthalpy known from literature[48]. Considering a glucose concentration of $5 \mathrm{mmol} / \mathrm{l}$, which is the highest in the linear range of Fig. 4D, the thus estimated highest consumption of glucose would be $14 \cdot 10^{-9} \mathrm{~mol} / \mathrm{min}$. Consequently, $14 \cdot 10^{-9} \mathrm{~mol} / \mathrm{min}$ of oxygen are also consumed, which is of the same order of magnitude as the initial DO moles available in solution $\left(7 \cdot 10^{-9} \mathrm{~mol}\right.$, assuming 0.25 $\mathrm{mM}$ as the initial DO concentration). We can conclude that the continuous replenishment of oxygen from air and the relatively fast diffusion of oxygen may explain the ability to test high glucose concentrations without oxygenlimiting effects. This is particularly true in the linear range of the Michaelis-Menten model, as no linearity between heat power signal and glucose concentration would have been observed if oxygen was limiting the reaction. In contrast, we cannot exclude that oxygen depletion may be partially taking place in the saturation regime of the Michaelis-Menten model (glucose concentration higher than $5 \mathrm{mmol} / \mathrm{l}$ ), even though the system follows the expected theoretical behaviour for such reaction. Finally, the combination of glucose oxidase with the oxygenregenerating enzyme catalase may be foreseen in future to create less limiting experimental conditions from the oxygen depletion point-of-view. 
The validation of the nanocalorimetric platform with this biochemical reaction demonstrates its applicability to the thermodynamic study of chemical reactions, by measuring the enthalpy change of reaction, but also to the kinetic study of biochemical and, in particular, enzymatic assays. The versatility of the platform makes it an excellent tool for studying unknown biochemical reactions and, more interestingly, the effects of chemical compounds on specific enzymatic reactions: the latest is particularly relevant in the field of drug design for drugs that are targeting enzymes, which are considered one of the major drug targets[52].

\section{Conclusions}

The development and the validation of a new nanocalorimetric platform has been presented and its applicability to the study of both the thermodynamics and kinetics of (bio-)chemical reactions has been demonstrated. In particular, the design of the platform allowed achieving high temperature stability in the mK range, a thermalization time of less than 30 minutes and a sensing response time of a few seconds. The platform is easy to use, as it does not require any specific sample preparation prior to the experiment and potentially any thermochemical reaction may be investigated. Sample and reagent solutions can either be preloaded in an on-chip reservoir or injected directly into the reservoir via an external thermalized conduct, allowing very versatile experimental protocol definitions. The good performance of the nanocalorimetric platform to measure thermodynamic properties, in particular the heat of mixing of a 1-propanol-water test solutions and the study of the enzymatic activity of glucose oxidase, was successfully demonstrated. The following advantages of our platform were identified: (i) a high power sensitivity, as heat power signals as small as a few $\mu \mathrm{W}$ were successfully detected, (ii) small sample volumes in the $\mu \mathrm{l}$ range, which is of benefit for testing chemical compounds which are expensive or limited in quantity, (iii) a short thermalization time, enabling fast turnover between two experiments, (iv) an adequate temporal resolution for the sensor response, enabling studying fast chemical reactions. At the present, the system relies on passive diffusive mixing of the small injected sample volume into the larger on-chip reservoir. Faster mixing is key to further improvement of the platform performance and this may be achieved by integrating a custom-designed microfluidic chip system with the sensor chip in future. The present platform design is already compatible with such an implementation.

Finally, the presented work not only shows a new highly performing calorimetric platform per se, but it also represents a significant advance in the field of more user-friendly and versatile technologies for biology. We believe our approach will be in favour of exploitation of a powerful sensing technique like calorimetry in advanced biology studies, and particularly in molecular biology. In particular, such system holds great potential in particularly interesting fields of applications, such as drug design, allowing to easily study the mechanism of action of specific compounds on enzymatic reactions, or in the field of fundamental research in molecular biology, where specific molecular pathways which are regulated by enzymes may be investigated.

\section{Acknowledgements}

This work was supported by the EPFL and funding was provided by the EU Ideas program (ERC-2012-AdG-320404). We also thank members of the EPFL workshops for printed circuit board fabrication and for micromechanics for the technical assistance.

\section{References}

[1] W. Lee, Lee, and Koh, "Development and applications of chip calorimeters as novel biosensors," Nanobiosensors Dis. Diagn., vol. 2012, pp. 17-29, Apr. 2012.

[2] J. B. Chaires, "Calorimetry and Thermodynamics in Drug Design," Annu. Rev. Biophys., vol. 37, no. 1, pp. 135$151,2008$. 
[3] A. E. Beezer, "An outline of new calculation methods for the determination of both thermodynamic and kinetic parameters from isothermal heat conduction microcalorimetry," Thermochim. Acta, vol. 380, no. 2, pp. 205-208, Dec. 2001.

[4] M. L. Bianconi, "Calorimetry of enzyme-catalyzed reactions," Biophys. Chem., vol. 126, no. 1-3, pp. 59-64, Mar. 2007.

[5] M. I. Recht, F. E. Torres, D. D. Bruyker, A. G. Bell, M. Klumpp, and R. H. Bruce, "Measurement of enzyme kinetics and inhibitor constants using enthalpy arrays," Anal. Biochem., vol. 388, no. 2, pp. 204-212, May 2009.

[6] A. E. Beezer, Biological microcalorimetry. London: Academic Press Inc. Ltd, 1980.

[7] I. Wadsö, "Bio-calorimetry," Trends Biotechnol., vol. 4, no. 2, pp. 45-51, Feb. 1986.

[8] O. Braissant, D. Wirz, B. Göpfert, and A. U. Daniels, "Use of isothermal microcalorimetry to monitor microbial activities," FEMS Microbiol. Lett., vol. 303, no. 1, pp. 1-8, 2010.

[9] O. Braissant, D. Wirz, B. Göpfert, and A. U. Daniels, "Biomedical Use of Isothermal Microcalorimeters," Sensors, vol. 10, no. 10, pp. 9369-9383, Oct. 2010.

[10] L. M. Ahmad, B. Towe, A. Wolf, F. Mertens, and J. Lerchner, "Binding event measurement using a chip calorimeter coupled to magnetic beads," Sens. Actuators B Chem., vol. 145, no. 1, pp. 239-245, Mar. 2010.

[11] I. Wadsö and L. Wadsö, "Systematic errors in isothermal micro- and nanocalorimetry</o:p>," J. Therm. Anal. Calorim., vol. 82, no. 3, pp. 553-558, Nov. 2005.

[12] T. Maskow, T. Schubert, A. Wolf, F. Buchholz, L. Regestein, J. Buechs, F. Mertens, H. Harms, and J. Lerchner, "Potentials and limitations of miniaturized calorimeters for bioprocess monitoring," Appl. Microbiol. Biotechnol., vol. 92, no. 1, pp. 55-66, Oct. 2011.

[13] J. Lerchner, A. Wolf, H.-J. Schneider, F. Mertens, E. Kessler, V. Baier, A. Funfak, M. Nietzsch, and M. Krügel, "Nano-calorimetry of small-sized biological samples," Thermochim. Acta, vol. 477, no. 1-2, pp. 48-53, Oct. 2008.

[14] F. Yi and D. A. La Van, "Nanoscale thermal analysis for nanomedicine by nanocalorimetry," Wiley Interdiscip. Rev. Nanomed. Nanobiotechnol., vol. 4, no. 1, pp. 31-41, 2012.

[15] A. W. van Herwaarden, "Overview of calorimeter chips for various applications," Thermochim. Acta, vol. 432, no. 2, pp. 192-201, Jul. 2005.

[16] V. Baier, R. Födisch, A. Ihring, E. Kessler, J. Lerchner, G. Wolf, J. M. Köhler, M. Nietzsch, and M. Krügel, "Highly sensitive thermopile heat power sensor for micro-fluid calorimetry of biochemical processes," Sens. Actuators Phys., vol. 123-124, pp. 354-359, Sep. 2005.

[17] E. A. Johannessen, J. M. R. Weaver, L. Bourova, P. Svoboda, P. H. Cobbold, and J. M. Cooper, "Micromachined Nanocalorimetric Sensor for Ultra-Low-Volume Cell-Based Assays," Anal. Chem., vol. 74, no. 9, pp. 2190-2197, May 2002.

[18] W. Lee, W. Fon, B. W. Axelrod, and M. L. Roukes, "High-sensitivity microfluidic calorimeters for biological and chemical applications," Proc. Natl. Acad. Sci. U. S. A., vol. 106, pp. 15225-30, 2009.

[19] S.-K. Nam, J.-K. Kim, S.-C. Cho, and S.-K. Lee, "Design and Characterization of a High Resolution Microfluidic Heat Flux Sensor with Thermal Modulation," Sensors, vol. 10, no. 7, pp. 6594-6611, Jul. 2010.

[20] L. Wang, D. M. Sipe, Y. Xu, and Q. Lin, "A MEMS Thermal Biosensor for Metabolic Monitoring Applications," J. Microelectromechanical Syst., vol. 17, no. 2, pp. 318-327, 2008.

[21] K. Ramanathan and B. Danielsson, "Principles and applications of thermal biosensors," Biosens. Bioelectron., vol. 16, no. 6, pp. 417-423, Aug. 2001.

[22] M. I. Recht, D. D. Bruyker, A. G. Bell, M. V. Wolkin, E. Peeters, G. B. Anderson, A. R. Kolatkar, M. W. Bern, P. Kuhn, R. H. Bruce, and F. E. Torres, "Enthalpy array analysis of enzymatic and binding reactions," Anal. Biochem., vol. 377, no. 1, pp. 33-39, Jun. 2008.

[23] F. E. Torres, P. Kuhn, D. D. Bruyker, A. G. Bell, M. V. Wolkin, E. Peeters, J. R. Williamson, G. B. Anderson, G. P. Schmitz, M. I. Recht, S. Schweizer, L. G. Scott, J. H. Ho, S. A. Elrod, P. G. Schultz, R. A. Lerner, and R. H. Bruce, "Enthalpy arrays," Proc. Natl. Acad. Sci. U. S. A., vol. 101, no. 26, pp. 9517-9522, Jun. 2004.

[24] B. Lubbers and F. Baudenbacher, "Isothermal Titration Calorimetry in Nanoliter Droplets with Subsecond Time Constants," Anal. Chem., vol. 83, no. 20, pp. 7955-7961, Oct. 2011.

[25] K. Verhaegen, K. Baert, J. Simaels, and W. Van Driessche, "A high-throughput silicon microphysiometer," Sens. Actuators Phys., vol. 82, no. 1-3, pp. 186-190, May 2000. 
[26] J. Xu, R. Reiserer, J. Tellinghuisen, J. P. Wikswo, and F. J. Baudenbacher, "A Microfabricated Nanocalorimeter: Design, Characterization, and Chemical Calibration," Anal. Chem., vol. 80, no. 8, pp. 2728-2733, Apr. 2008.

[27] E. A. Johannessen, J. M. R. Weaver, P. H. Cobbold, and J. M. Cooper, "A Suspended Membrane Nanocalorimeter for Ultralow Volume Bioanalysis," IEEE Trans. NANOBIOSCIENCE, vol. 1, no. 1, pp. 29-36, Mar. 2002.

[28] E. A. Johannessen, J. M. R. Weaver, P. H. Cobbold, and J. M. Cooper, "Heat conduction nanocalorimeter for pl-scale single cell measurements," Appl. Phys. Lett., vol. 80, no. 11, pp. 2029-2031, Mar. 2002.

[29] B. S. Kwak, B. S. Kim, H. H. Cho, J. S. Park, and H. I. Jung, "Dual thermopile integrated microfluidic calorimeter for biochemical thermodynamics," Microfluid. Nanofluidics, vol. 5, no. 2, pp. 255-262, Aug. 2008.

[30] B. Wang and Q. Lin, "Temperature-modulated differential scanning calorimetry in a MEMS device," Sens. Actuators B Chem., vol. 180, pp. 60-65, Apr. 2013.

[31] Y. Zhang and S. Tadigadapa, "Calorimetric biosensors with integrated microfluidic channels," Biosens. Bioelectron., vol. 19, no. 12, pp. 1733-1743, Jul. 2004.

[32] J. Lerchner, A. Wolf, G. Wolf, and I. Fernandez, "Chip calorimeters for the investigation of liquid phase reactions: Design rules," Thermochim. Acta, vol. 446, no. 1-2, pp. 168-175, Jul. 2006.

[33] J. Lerchner, A. Wolf, G. Wolf, V. Baier, E. Kessler, M. Nietzsch, and M. Krügel, "A new micro-fluid chip calorimeter for biochemical applications," Thermochim. Acta, vol. 445, no. 2, pp. 144-150, Jun. 2006.

[34] J. Lerchner, A. Wolf, F. Buchholz, F. Mertens, T. R. Neu, H. Harms, and T. Maskow, "Miniaturized calorimetry - A new method for real-time biofilm activity analysis," J. Microbiol. Methods, vol. 74, no. 2-3, pp. 74-81, Aug. 2008.

[35] J. Lerchner, D. Mueller-Hagen, H. Roehr, A. Wolf, F. Mertens, R. Mueller, W. Witte, and I. Klare, "Chipcalorimetric evaluation of the efficacy of antibiotics and bacteriophages against bacteria on a minutetimescale," J. Therm. Anal. Calorim., vol. 104, no. 1, pp. 31-36, Apr. 2011.

[36] T. Maskow, J. Lerchner, M. Peitzsch, H. Harms, and G. Wolf, "Chip calorimetry for the monitoring of whole cell biotransformation," J. Biotechnol., vol. 122, no. 4, pp. 431-442, Apr. 2006.

[37] T. Hartmann, N. Barros, A. Wolf, C. Siewert, P. L. O. Volpe, J. Schemberg, A. Grodrian, E. Kessler, F. Hänschke, F. Mertens, and J. Lerchner, "Thermopile chip based calorimeter for the study of aggregated biological samples in segmented flow," Sens. Actuators B Chem., vol. 201, pp. 460-468, Oct. 2014.

[38] A. Wolf, T. Hartmann, M. Bertolini, J. Schemberg, A. Grodrian, K. Lemke, T. Förster, E. Kessler, F. Hänschke, F. Mertens, R. Paus, and J. Lerchner, "Toward high-throughput chip calorimetry by use of segmented-flow technology," Thermochim. Acta, vol. 603, pp. 172-183, Mar. 2015.

[39] I. Wadso and R. N. Goldberg, "Standards in isothermal microcalorimetry (IUPAC Technical Report)," Pure Appl. Chem., vol. 73, no. 10, pp. 1625-1639, 2001.

[40] M. I. Davis and E. S. Ham, "Analysis and interpretation of excess molar properties of amphiphile + water systems: Part 2. Comparisons of the propanol isomers in their aqueous mixtures," Thermochim. Acta, vol. 190, no. 2, pp. 251-258, Nov. 1991.

[41] M. F. Chaplin and C. Bucke, Enzyme Technology. Cambridge: CUP Archive, 1990.

[42] A. Crueger and W. Crueger, "Glucose Transforming Enzymes," in Microbial Enzymes and Biotechnology, Second., W. M. Fogarty and C. T. Kelly, Eds. Essex: Elsevier Science Publishers, 1990, pp. 177-226.

[43] D. Kondepudi and I. Prigogine, "Thermodynamics of Solutions," in Modern Thermodynamics, John Wiley \& Sons, Ltd, 2014, pp. 207-229.

[44] J. M. Berg, J. L. Tymoczko, and L. Stryer, Biochemistry: International Edition, International, of 6th revised. New York: W.H.Freeman \& Co Ltd, 2006.

[45] K. A. Johnson and R. S. Goody, "The Original Michaelis Constant: Translation of the 1913 Michaelis-Menten Paper," Biochemistry (Mosc.), vol. 50, no. 39, pp. 8264-8269, Oct. 2011.

[46] L. Michaelis and M. L. Menten, "Die Kinetik der Invertinwirkung," Biochem. Z., vol. 49, pp. 333-369, 1913.

[47] S. B. Bankar, M. V. Bule, R. S. Singhal, and L. Ananthanarayan, "Glucose oxidase - An overview," Biotechnol. Adv., vol. 27, no. 4, pp. 489-501, Jul. 2009.

[48] R. Hüttl, K. Bohmhammel, K. Pritzkat, and G. Wolf, "Problems associated with using thermal measurement principles in enzymatic reactions," Thermochim. Acta, vol. 229, pp. 205-213, Dec. 1993.

[49] R. Wilson and A. P. F. Turner, "Glucose oxidase: an ideal enzyme," Biosens. Bioelectron., vol. 7, no. 3, pp. 165185, 1992. 
[50] B. Danielsson, K. Gadd, B. Mattiasson, and K. Mosbach, "Enzyme thermistor determination of glucose in serum using immobilized glucose oxidase," Clin. Chim. Acta, vol. 81, no. 2, pp. 163-175, Dec. 1977.

[51] P. Han and D. M. Bartels, "Temperature Dependence of Oxygen Diffusion in H2O and D2O," J. Phys. Chem., vol. 100, no. 13, pp. 5597-5602, Jan. 1996.

[52] J. G. Robertson, "Mechanistic Basis of Enzyme-Targeted Drugs," Biochemistry (Mosc.), vol. 44, no. 15, pp. 5561-5571, Apr. 2005. 

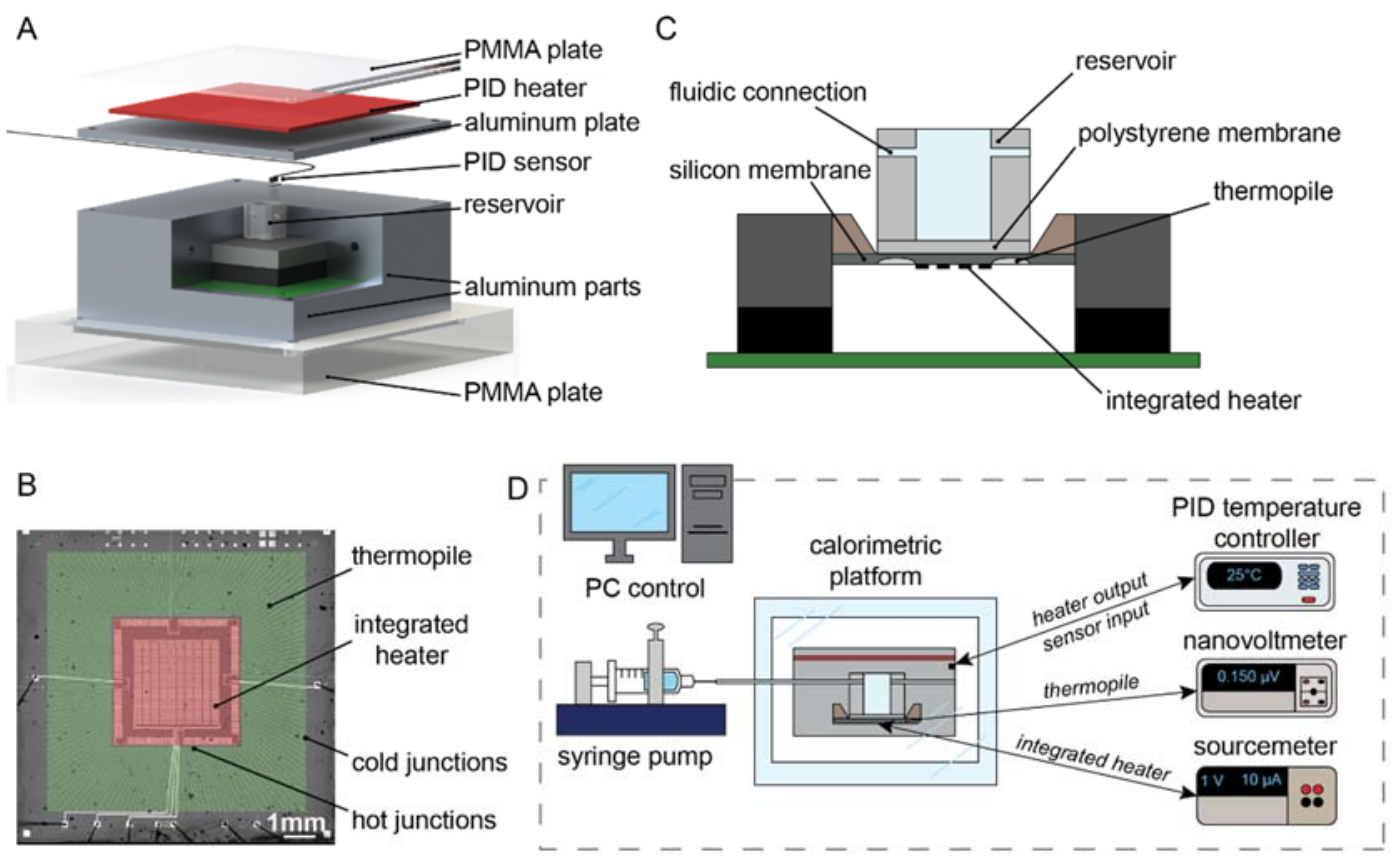

Fig 1 Overview of the full nanocalorimetric platform. A) Three-dimensional sketch of the isothermal holder, consisting mainly of a $8.3 \times 8.3 \mathrm{~mm}^{2}$ silicon membrane-based thermopile sensor, a PC sample reservoir positioned on the sensor membrane, and several aluminum parts clamped together (outer dimensions $60 \times 60 \mathrm{~mm}^{2}$ ). The PID temperature control is achieved thanks to a flat polyimide heater (PID heater) and a platinum temperature sensor (PID sensor), which are both positioned inside the isothermal holder. B) Top view of the nanocalorimetric chip (XENNCM 9924, Xensor Integration), indicating the position of hot and cold junctions of the silicon-aluminum thermopile (highlighted in green) and of the integrated aluminum heaters (highlighted in red) on the monocrystalline silicon membrane. C) Cross-sectional view of the core of the platform. The $25 \mu \mathrm{m}$ polystyrene membrane at the bottom of sample reservoir is in direct contact with the sensor membrane. Fluidic connections are fabricated on the sidewalls of the reservoir and aligned to the corresponding connections in the aluminum part providing direct access from the outside. D) Different elements of the nanocalorimetric platform for fluidic sample injection, electrical signal acquisition and temperature control. The control of the full system is automatized through a Labview (National Instruments) interface. 

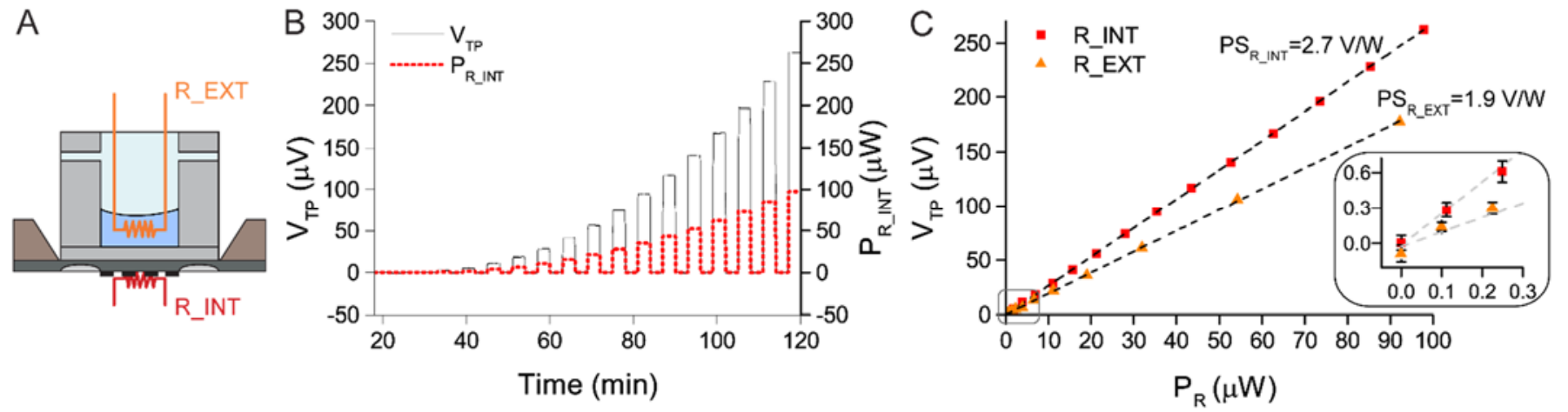

Fig 2 Thermo-electrical calibration of the nanocalorimetric platform at $25^{\circ} \mathrm{C}$. A) Sketch of the reservoir on the silicon membrane, showing the two configurations used for the calibration: (i) the resistive thin-film heater integrated directly on the silicone membrane $\left(R \_I N T\right)$, or (ii) a miniaturized $100 \mathrm{k} \Omega$ thermistor used as resistive heater, inserted into the reservoir filled with deionized water $\left(R_{-} E X T\right)$. B) Thermopile voltage $\left(V_{T P}\right)$ over time curve (full line), when applying a periodically increasing electrical power to the integrated resistive heater $P_{R_{\_} I N T}$ (dashed line). C) Comparison of the calibration curve based on the integrated resistive heater $R_{-} I N T$ and on the external resistive heater $R_{-} E X T$. The corresponding power sensitivities are $2.7 \mathrm{~V} / \mathrm{W}\left(P S_{R_{-} I N T}\right)$ and $1.9 \mathrm{~V} / \mathrm{W}\left(P S_{R_{-} E X T}\right)$, respectively. The inset is a close-up of the calibration points for very small heat power $(0-300 \mathrm{nW})$ that allow to determine the limit of detection of $70 \mathrm{nW}$ and $170 \mathrm{nW}$, when using $R_{-} I N T$ or $R_{-} E X T$, respectively. 

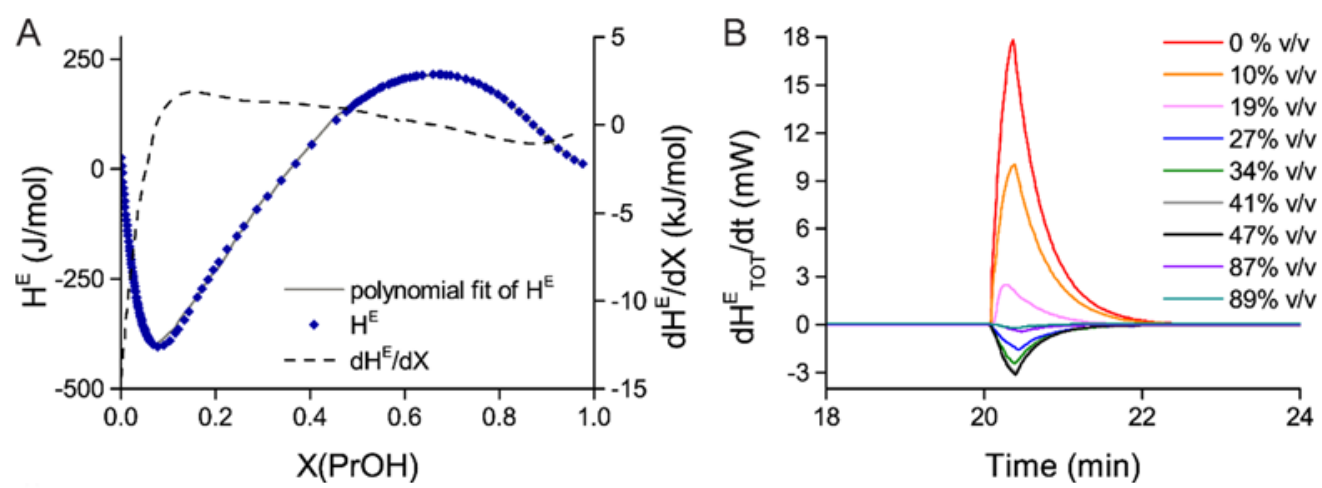

$c$

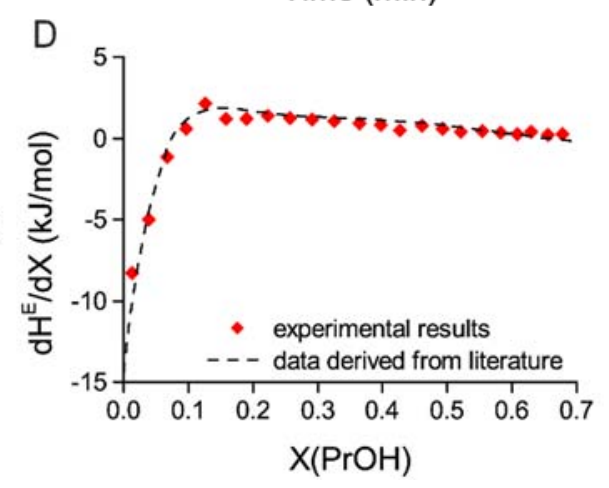

Fig 3 Measurements of the heat of mixing of 1-propanol in water at $25^{\circ} \mathrm{C}$. A) Literature data for the heat of mixing per mole (or molar excess enthalpy) HE of 1-propanol in water vs the mole fraction $\mathrm{X}(\mathrm{PrOH})$ of 1-propanol in water[40]. Additionally, the differential molar excess enthalpy $\mathrm{dHE} / \mathrm{dX}$ is shown, obtained by differentiating the polynomial fit of the molar excess enthalpy $\mathrm{HE}$ with respect to the mole fraction $\mathrm{X}(\mathrm{PrOH})$ (dashed line). B) Differential excess enthalpy with respect to time dHETOT/dt generated by injecting and mixing $5 \mathrm{~L}$ of pure 1propanol into $45 \mathrm{~L}$ of 1-propanol solutions at different initial concentrations. The differential excess enthalpy $\mathrm{dHETOT/dt} \mathrm{corresponds} \mathrm{to} \mathrm{the} \mathrm{heat} \mathrm{power} \mathrm{detected} \mathrm{by} \mathrm{the} \mathrm{thermopile} \mathrm{and} \mathrm{it} \mathrm{was} \mathrm{obtained} \mathrm{by} \mathrm{measuring} \mathrm{the}$ thermopile voltage VTP and applying a power sensitivity coefficient of $0.6 \mathrm{~V} / \mathrm{W}$. C) Procedure used for data analysis: (i) the measured value dHETOT/dt is first normalized (dividing by the number of moles in the mixture) to obtain the differential molar excess enthalpy dHE/dt. (ii) Integrating over time and dividing by the mole fraction difference (Xf - $\mathrm{X}$, after and before injection) results in the differential molar excess enthalpy $\mathrm{dHE} / \mathrm{dX}$ with respect to the mole fraction $X$. (iii) The central mole fraction $X c$ is calculated as the average mole fraction between the initial (Xi) and final (Xf) mole fraction and is used to plot the differential molar excess enthalpy data points (Xc, dHE/dX). D) Comparison of the differential molar excess enthalpy $\mathrm{dHE} / \mathrm{dX}$ derived from literature[40] with the values calculated from our experimental data. 

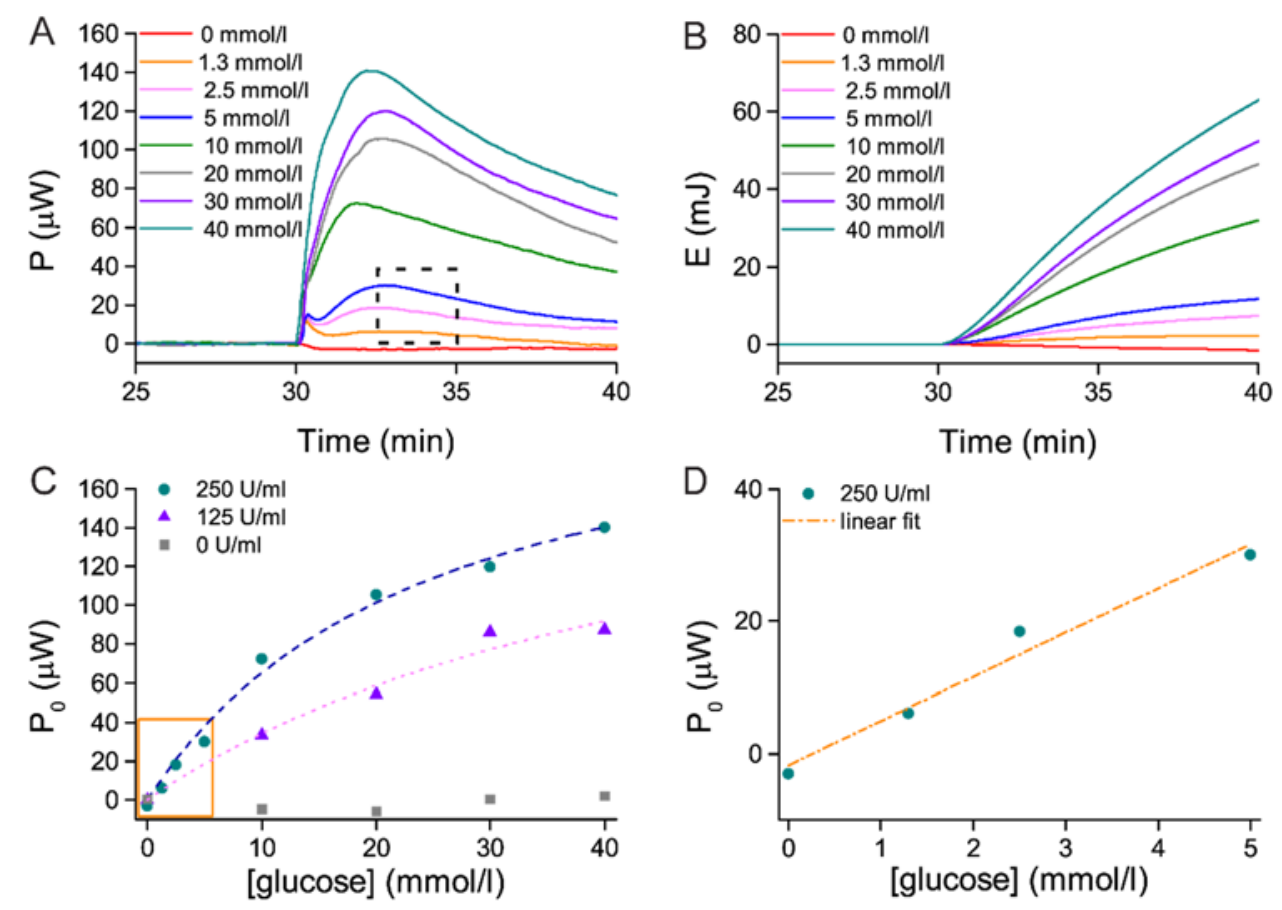

Fig 4 Study of glucose oxidation, catalyzed by glucose oxidase at $25^{\circ} \mathrm{C}$. A) Heat power $P$ measured over time, as obtained from the thermopile voltage $V_{T P}$ assuming a power sensitivity coefficient of $1 \mathrm{~V} / \mathrm{W}$. The reservoir is initially filled with $25 \mu \mathrm{l}$ of glucose oxidase solution (250 U/ml in PBS) and $1.5 \mu$ of glucose solution (in PBS with varying concentrations) is injected at $\mathrm{t}=30 \mathrm{~min}$ to achieve a final glucose concentration ranging from 0 to $40 \mathrm{mmol} / \mathrm{l}$. $\mathrm{B}$ ) Thermal energy $E$ released during the reaction, obtained by integrating $P$ with respect to time, for different glucose solutions and at the fixed glucose oxidase concentration of $250 \mathrm{U} / \mathrm{ml}$. C) Maximum measured heat power $P_{0}$ plotted as a function of glucose concentration, for different glucose oxidase concentrations: $250 \mathrm{U} / \mathrm{ml}, 125 \mathrm{U} / \mathrm{ml}$ and 0 $\mathrm{U} / \mathrm{ml}$ (control experiment). The experimental data obtained for $250 \mathrm{U} / \mathrm{ml}$ and $125 \mathrm{U} / \mathrm{ml}$ of glucose oxidase were fitted using the Michaelis-Menten equation as shown by the dashed lines (adjusted R-squared $\approx 0.99$ and 0.97 respectively). D) Magnification of the maximum heat power $P_{0}$ vs glucose concentration curve for the glucose oxidase concentration of $250 \mathrm{U} / \mathrm{ml}$ (from Fig. 4C). This emphasizes the linear region, typical of a Michaelis-Menten curve for glucose concentrations smaller than the Michaelis constant $K_{M}$. In this case, the linear region is observed for glucose concentrations below $10 \mathrm{mmol} / \mathrm{l}$. A linear fitting (dashed line, adjusted R-squared $\approx 0.96$ ) allows the estimation of the proportionality coefficient $\alpha \approx 6.68 \mu \mathrm{W} /(\mathrm{mmol} / \mathrm{l})$. 